\title{
Initial cervical dilation and the association of the success of atosiban
}

\begin{abstract}
Objectives: This research aims at studying the impact of initial cervical dilation on the Atosiban efficacy in terms of prolongation of pregnancy.

Methodology: A retrospective observational study of 84 pregnant women admitted to the hospital with a diagnosis of threatened preterm labor, and who received Atosiban as a tocolytic for prevention of preterm delivery (suppression of uterine contractions) in the period 1st January 2015 till 31st December 2015 and their follow up till the timing of their delivery, data were collected using an online data collecting system through hospital online recording system. The inclusion criteria included pregnant women aged between 18 years and 35 years old in whom gestational age ranged between 24 weeks and 33 weeks and 6 days.
\end{abstract}

Results: Total of 84 cases were studied. Mean cervical dilatation at presentation was $(1,9 \longleftarrow 0,25 \mathrm{~cm})$ with median of $2 \mathrm{~cm}$. Two groups were made according to cervical dilation $(<2$ and $\geq 2 \mathrm{~cm})$. No significant deference between both groups for maternal age [p value 0,59], parity [p value of 0.41 ], Gestational age at preterm delivery diagnosis [p value 0.49], and Duration of the Atosiban [p value 0,54]. Atosiban StopDelivery interval was significantly deferred among the two groups [p value of 0,003 ] as well as the gestational age at delivery $[0,039]$. Mean gestational age at delivery was 35 weeks (SD 1,3) and 33,2 weeks (SD 1,1) for cervical dilation $<2$ and $\geq 2$ respectively $[\mathrm{P}=0,039] ; 54 \%$ and $45 \%$ both groups respectively had Atosiban for 48 hours. $22,5 \%$ and $46 \%$ of both groups respectively delivered within 7 days of the diagnosis $(\mathrm{p}=0,034)$.

Conclusion: Our results showed that the Initial cervical dilatation has a significant impact on the success of Atosiban' hence the prolongation of pregnancy. Such information is essential for decision-makers for a proactive approach.
Volume 5 Issue 2 - 2019

\section{Mohamed A Khalil, Rabe'a Saad, Abdullah Al Ibrahim}

Department of Obstetrics and Gynaecology, Women Wellness and Research Centre, Qatar

Correspondence: Mohamed A Khalil, Department of Obstetrics and Gynaecology, Women Wellness and Research Centre, Hamad Medical Corporation, Doha, Qatar, Emailmohdoc_1985@hotmail.com

Received: April 08, 2019 | Published: April 23, 2019

\section{Introduction}

Preterm birth is defined as delivery at less than 37 weeks gestation and is the leading cause of perinatal morbidity and mortality. ${ }^{1}$ Preterm birth (birth occurring before 37 weeks of gestation) is a global public health challenge, with 15 million infants born preterm every year and an estimated $35 \%$ of deaths in the first 4 weeks of life are directly attributable to prematurity. ${ }^{2}$ In 2013 , preterm birth complications were the leading cause of death of the estimated 6.3 million liveborn children worldwide; Thus, research studies have been focused on identifying the best method to prevent preterm birth. ${ }^{3}$ Atosiban, an oxytocin receptor blocker which has been used in our institute, in cases of suspected preterm labor for thirteen years. This research aims at studying Atosiban efficacy in terms of prolongation of pregnancy taking the initial cervical dilation (upon admission) is the principle factor.

\section{Methodology}

A retrospective observational study where the data of 84 women were collected in the period from 1st January 2015 till 31st December 2015. All were admitted with the diagnosis of threatened preterm labor, and received Atosiban as a tocolysis to suppress uterine contractions in the period. The data of the follow up till delivery were collected retrospectively using an online data collecting system through the hospital Clinical Information System [Cerner; version 12.4.0 (441592)-2016 Citrix Systems, Inc].

The inclusion criteria included pregnant women with reproductive age group 18 years old till 35 years old whom gestation age ranged between 24 weeks till 33 weeks and 6 days. Pregnant women with following criteria were excluded from the study; reproductive age group less than 18 years old or more than 35 years old, gestational age less than 24 weeks or 34 weeks and more, cervical dilatation $5 \mathrm{~cm}$ or more on admission, cervical dilatation $4 \mathrm{~cm}$ and effacement $100 \%$ on admission, severe vaginal bleeding, signs of fetal distress, signs of intrauterine infection, and fetuses with suspected chromosomal abnormalities or anomalies incompatible with life. Atosiban was given as a single loading intravenous dose, $6.75 \mathrm{mg}$ in $0.9 \%$ sodium chloride solution, followed by an intravenous infusion of 300 micrograms/ min in $0.9 \%$ sodium chloride solution for the first 3 hours and then $100 \mathrm{micrograms} / \mathrm{min}$ for another 45 hours. Assigned treatment was planned for up to 48 hours. Maintenance therapy after the first 48 hours was not provided. Prophylactic antibiotics for group B streptococcus and corticosteroids were administered according to standard clinical indications. The primary outcome was to assess the effect of initial cervical dilation on the success of Tocolytic using Atosiban. We created two groups $(<2 \mathrm{~cm}$ and $\geq 2 \mathrm{~cm})$ and Deferent variables were studied, maternal age, parity, number of fetuses, duration of Atosiban therapy, the length of period after stopping Atosiban and delivery as well as 
the gestation age at delivery. The data were kept anonymously in an Excel sheet [(Microsoft Office -2016 (16.0.6769.2017)]. Descriptive and inferential statistics were employed as adequate using Wizard Pro (version 1.9.26). The statically differences were calculated using the t-test for the means and the Mann-Whitney test for the medians. A P-value of less than 0.05 was considered statistically significant. Ethical approval was not required as this was an audit and all data were unidentifiable.

\section{Results}

During the study period (Graph 1), a Total of 84 cases were studied. There is a increment in the rate of PL as the age goes up that could be explained by mean maternal age of 27years and graph 3 of parity could help understand that as half of our cohort are primigravida of whom $45 \%$ are above $25 y$ ears, para 1 and 2 comprise $40 \%$ of the PL patient and in both groups $>80 \%$ are above 25 years. Mean cervical dilatation at presentation was $(1,9 \pm 0,25 \mathrm{~cm})$ with median of $2 \mathrm{~cm}$. Two groups were made according to cervical dilation $(<2$ and $\geq 2 \mathrm{~cm})$ (Graph 2) (Table 1).

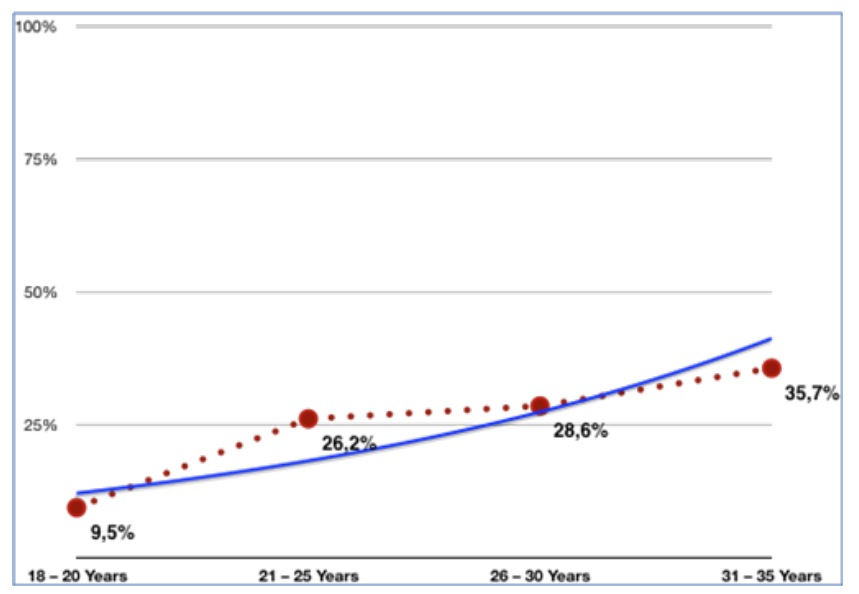

Graph I Maternal age distribution.

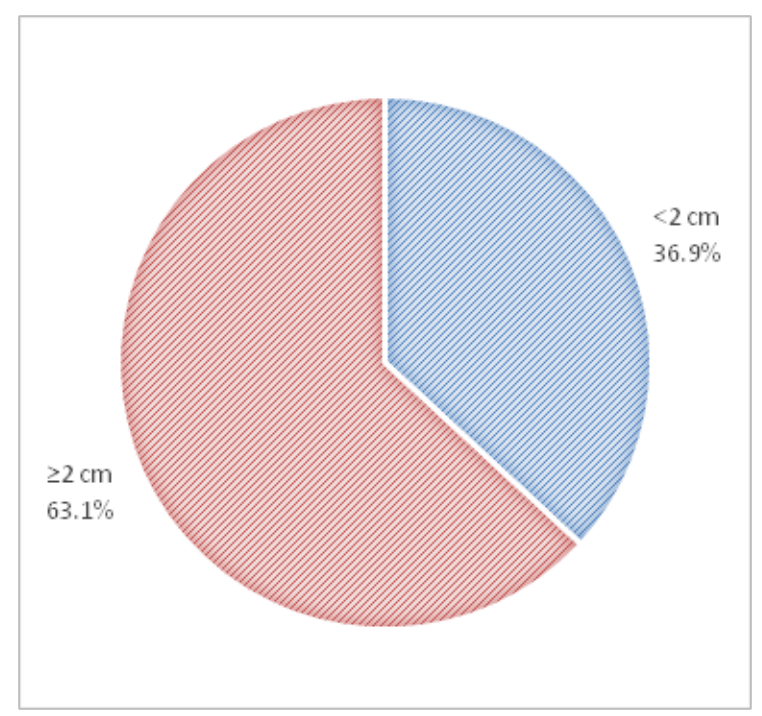

Graph 2 Two groups were made according to cervical dilation ( $<2$ and $\geq 2$ $\mathrm{cm})$.
Table I The biodata and clinical variables among both groups

\begin{tabular}{|c|c|c|c|c|}
\hline Variable & $<2 \mathrm{~cm}$ & $\geq 2 \mathrm{~cm}$ & $\begin{array}{l}P \\
\text { value }\end{array}$ & Significance \\
\hline $\begin{array}{l}\text { Maternal Age } \\
(\text { Mean } \pm \text { SD) years }\end{array}$ & $27,8 \pm 1,8$ & $27,2 \pm 1,3$ & 0,59 & NS \\
\hline Gravida (Median) & 2 & 2 & 0,69 & NS \\
\hline Para (Median) & 0 & I & 0,41 & NS \\
\hline $\begin{array}{l}\text { Gestational age at } \\
\text { Diagnosis (Mean } \pm S D) \\
\text { Weeks }\end{array}$ & $30,5 \pm 0,98$ & $30,9 \pm 0,7$ & 0,49 & NS \\
\hline 24-32 Weeks (\%) & $58,1 \%$ & $49,1 \%$ & 0,43 & NS \\
\hline 32-34 Weeks (\%) & $41,9 \%$ & $50,9 \%$ & 0,43 & NS \\
\hline $\begin{array}{l}\text { Atosiban Duration } \\
(\text { Mean } \pm S D) \text { Hours }\end{array}$ & $35,5 \pm 5,96$ & $\begin{array}{l}34,6 \\
\pm 4,4\end{array}$ & 0,82 & NS \\
\hline $\begin{array}{l}\text { Atosiban Stop- } \\
\text { Delivery Interval } \\
\text { (MeanSD) Days }\end{array}$ & $31,4 \pm 10,2$ & $16,2 \pm 5$ & 0,003 & S \\
\hline $\begin{array}{l}\text { Gestational Age at } \\
\text { Delivery (Mean } \pm S D) \\
\text { Weeks }\end{array}$ & $35 \pm 1,3$ & $\begin{array}{l}33,2 \\
\pm I, I\end{array}$ & 0,039 & S \\
\hline $\begin{array}{l}\text { Delivered within } 48 \\
\text { Hours (\%) }\end{array}$ & $19,4 \%$ & $26 \%$ & 0,49 & NS \\
\hline $\begin{array}{l}\text { Delivered within } 7 \\
\text { days (\%) }\end{array}$ & $22,5 \%$ & $46 \%$ & 0,034 & S \\
\hline
\end{tabular}

There was no significant defence regarding parity among the two groups [p value of 0.86], even taking each para and compare both groups, there was no significant defence (Graphs 3-9). Mean gestational age at delivery was 35 weeks (SD 1,3) and 33,2 weeks (SD $1,1)$ for cervical dilation $<2$ and $\geq 2$ respectively [ $\mathrm{P}=0,039]$; $54 \%$ and $45 \%$ both groups respectively had Atosiban for 48 hours. 19,4\% and $26 \%$ of both groups $(<2 \mathrm{~cm}$ and $\geq 2 \mathrm{~cm}$ respectively) delivered in the 1 st 48 hours $(0,49)$ see the graph for the Preterm delivery diagnosisdelivery interval. $22,5 \%$ and $46 \%$ of both groups respectively delivered within 7 days of the diagnosis $(p=0,034)$.

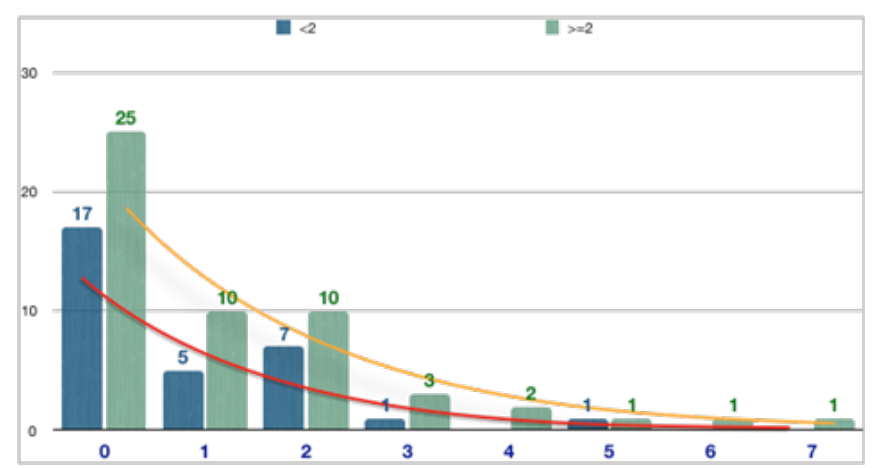

Graph 3 Parity categories. 


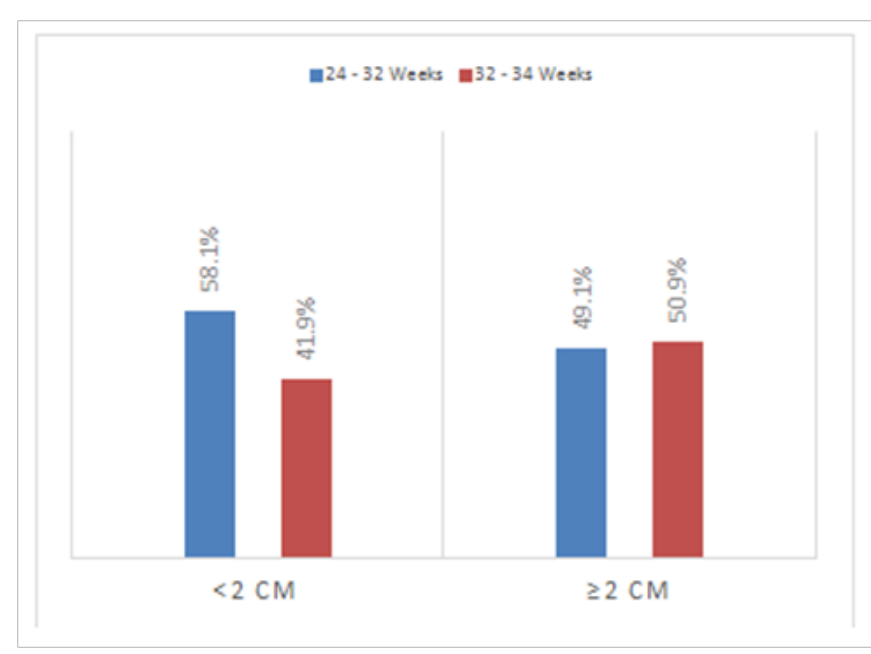

Graph 4 Gestational ranges among the two groups.

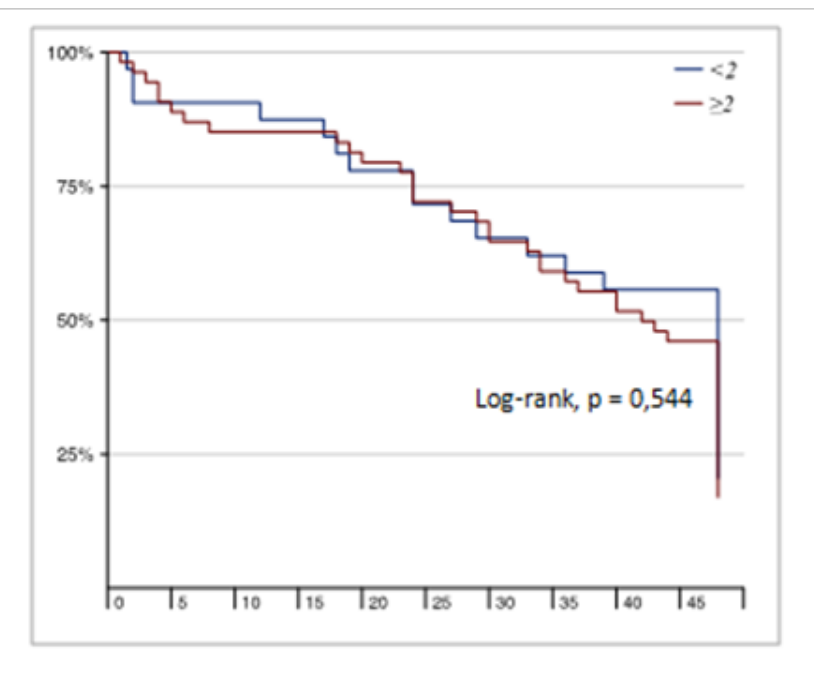

Graph 5 Kaplan-Meier Estimator Of The Duration Of The Atosiban.

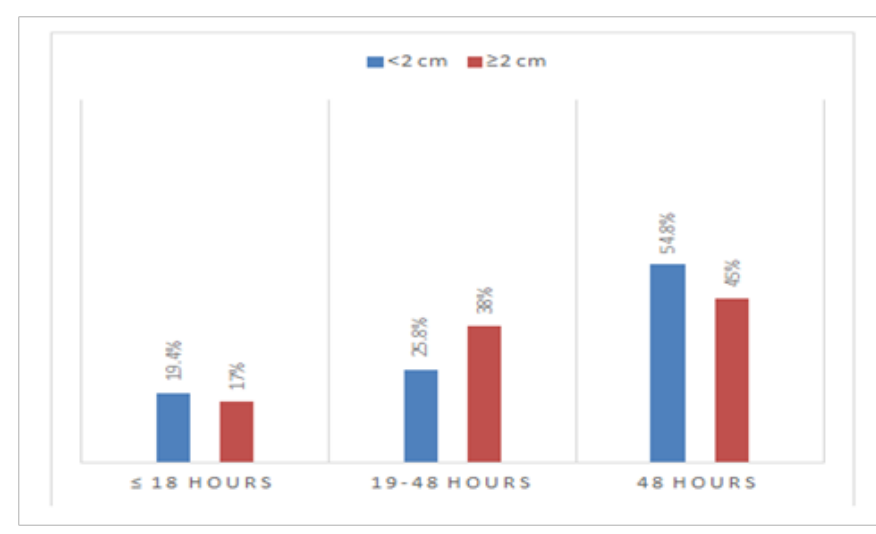

Graph 6 Duration of the Atosiban.

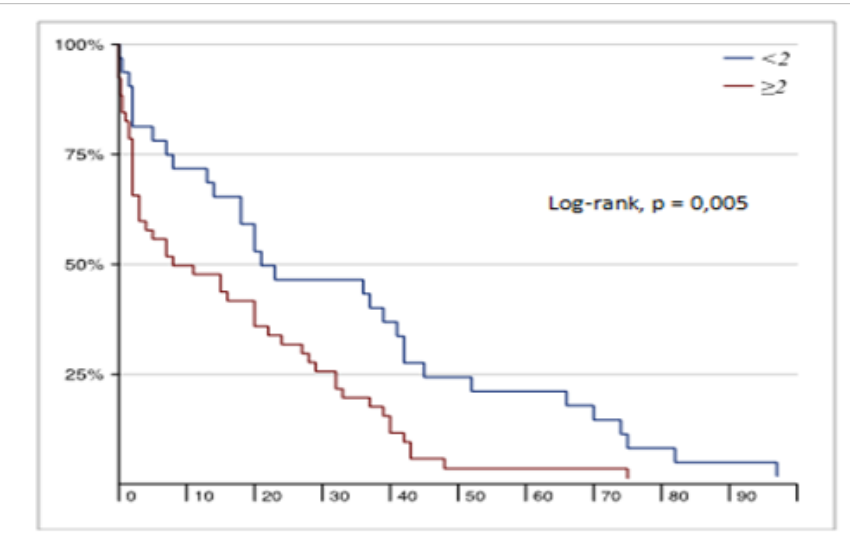

Graph 7 Kaplan-Meier Estimator OfThe Interval Between Stopping Atosiban And Delivery.

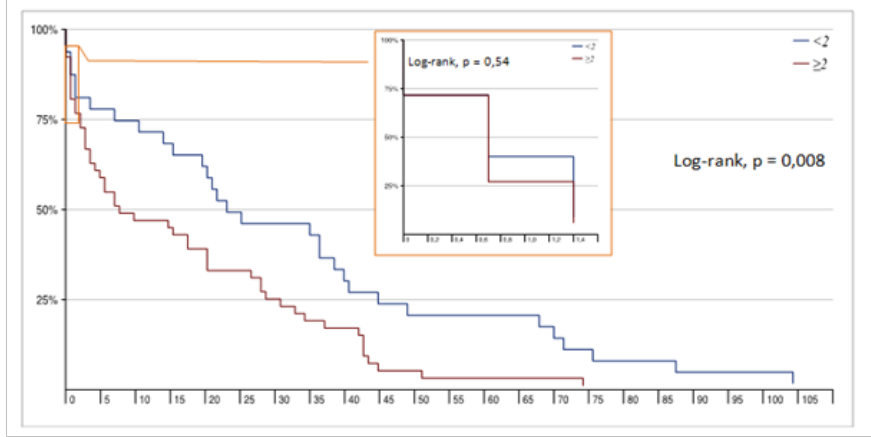

Graph 8 Ptl Diagnosis To Delivery Interval (Days).

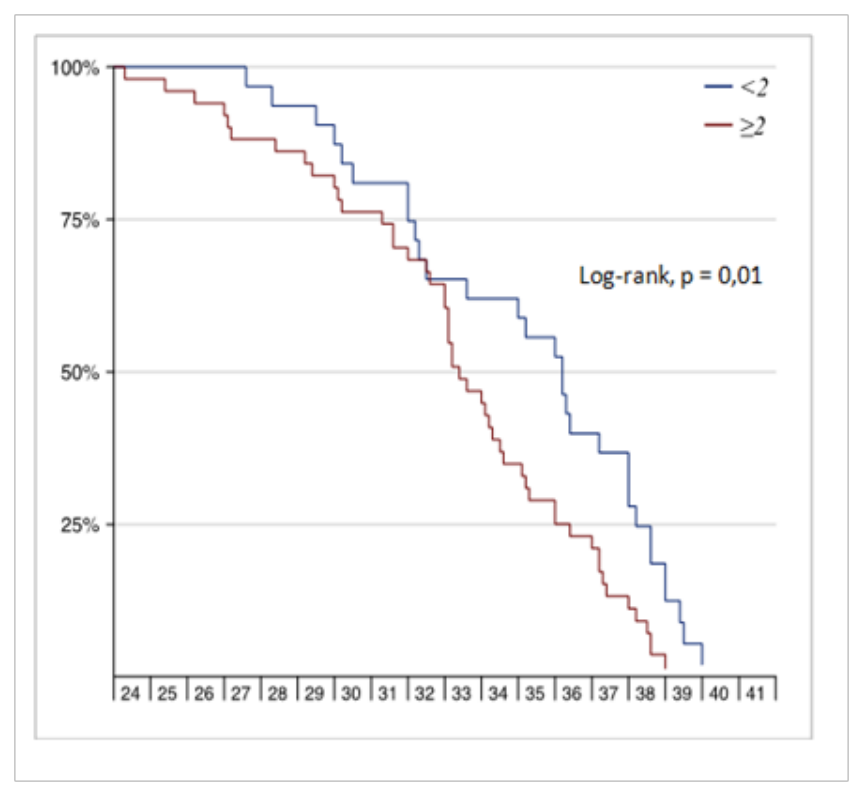

Graph 9 Gestational Age At Delivery (Weeks). 


\section{Discussion}

Atosiban, a novel parenteral agent used for treatment of acute PTL, is a competitive antagonist of oxytocin which blocks the surface oxytocin receptor of the myocyte. ${ }^{4}$

In women with threatened preterm birth, delay of delivery by 48 $\mathrm{h}$ allows antenatal corticosteroids to improve neonatal outcomes, for this reason tocoloysis suppose to be given for 48 hours. ${ }^{5}$ Our research demonstrate that almost half of the cohorts were given up Atosiban up to 48 hours, for the remaining, some delivered within 24 hours (31\%), as for the remaining the contractions settled down and the Atosiban stopped before 48 hours. In the meta-analysis of Haas et al. from $2009,{ }^{6}$ which included 1249 pregnant women, prolongation of pregnancy by 48 hours was achieved in $86 \%$ of cases and by 7 days in $78 \%$ of cases. Compared to our results of $77,3 \%$ in our cohort who continue beyond 48 hours and $64,3 \%$ beyond 7 days. There is a significant difference in both cohorts ( 0,03 and p 0,003 for 48 hours and seven days respectively), this can be explained by the high number of patients in Haas study, the other fact is the presence of $63 \%$ of our cohort who came with cervical dilatation $>2 \mathrm{~cm}$. It is of important notice that there was no significant difference among the two groups on the maternal age, parity, gestational age at diagnosis as well as Atosiban duration, yet cervical dilatation at diagnosis played a major rule on the success of Atosiban for the prolongation of pregnancy.

This highlight the fact that preterm parturition syndrome is complex and is initiated by multiple mechanisms, ${ }^{7}$ as we showed in our study; those beyond $2 \mathrm{~cm}$ were in the realm of such syndrome beyond the ability of Atosiaban of merely antagonise the oxytocin. Although our results have some good $p$ values for significance, hence the results might be used for counselling and anticipating further management; however, the small number of the cohort urge to expand it to involve a more cases in a preferable prospective manner.

\section{Conclusion}

Our results showed that the Initial cervical dilatation has a significant impact on the success of Atosiban' hence the prolongation of pregnancy. Such information is essential for decision-makers for a proactive approach (counselling, Neonatology involvement and plan delivery logistics, especially for those who need cesarean). Further study in this matter is required with more number of patients and prospective researches.

\section{Acknowledgments}

There is no financial interest associated with the study.

\section{Conflicts of interests}

There is no conflict of interest present in the realization of this clinical case.

\section{References}

1. Romero R, Sibai BM, Sanchez-Ramos L, et al. An oxytocin receptor antagonist (Atosiban) in the treatment of preterm labor: a randomized, doubleblind, placebo-controlled trial with tocolytic rescue. Am J Obstet Gynecol. 2000;182:1173-1183.

2. Blencowe H, Cousens S, Oestergaard MZ, et al. National, regional and worldwide estimates of preterm birth rates in the year 2010 with time trends for selected countries since 1990: a systematic analysis and implications. Lancet. 2012;379(9832):2162-2172.

3. Liu L, Johnson HL, Cousens S, et al. Global, regional, and national causes of child mortality in 2000-2010: an updated systematic analysis. Lancet. 2012;379(9832):2151-2161.

4. Groom KM. Preterm labour. Pharmacological prevention of prematurity. Best Pract Res Clin Obstet Gynaecol. 2007;21:843-856.

5. van Vliet EOG, Nijman Taj, Schuit E, et al. Nifedipine versus atosiban for threatened preterm birth (APOSTEL III): a multicentre, randomised controlled trial. Lancet, 2016;387(10033):2117-2124.

6. Haas DM, Imperiale TF, Kirkpatrick PR, et al. Tocolytic therapy: a metaanalysis and decision analysis. Obstet Gynecol. 2009;113:585-594.

7. Romero R, Espinoza J, Kusanovich J, et al. The preterm parturition syndrome. BJOG. 2006;113(Suppl 3):17-42. 